\title{
Meta
}

Journal des traducteurs

Translators' Journal

\section{SAGER, Juan C. (1990): A Practical Course in Terminology Processing, Amsterdam-Philadelphia, John Benjamins Publishing Company, XI + 254 p.}

\section{Jean-Claude Boulanger}

Volume 37, numéro 3, septembre 1992

URI : https://id.erudit.org/iderudit/002118ar

DOI : https://doi.org/10.7202/002118ar

Aller au sommaire du numéro

Éditeur(s)

Les Presses de l'Université de Montréal

ISSN

0026-0452 (imprimé)

1492-1421 (numérique)

Découvrir la revue

Citer ce compte rendu

Boulanger, J.-C. (1992). Compte rendu de [SAGER, Juan C. (1990) : A Practical Course in Terminology Processing, Amsterdam-Philadelphia, John Benjamins Publishing Company, XI + 254 p.] Meta, 37(3), 543-548.

https://doi.org/10.7202/002118ar d'utilisation que vous pouvez consulter en ligne.

https://apropos.erudit.org/fr/usagers/politique-dutilisation/ 


\section{Comptes rendus}

SAGER, Juan C. (1990): A Practical Course in Terminology Processing, AmsterdamPhiladelphia, John Benjamins Publishing Company, XI + 254 p.

Ce livre rassemble et analyse un certain nombre de questions fondamentales en matière de terminologie. L'auteur y scrute les différentes perspectives historiques et contemporaines qui éclairent ses positions actuelles et qui façonnent son credo scientifique. Je les schématise rapidement et de manière non exclusive car elles seront reprises ciaprès dans le détail des chapitres : en tant que discipline, la terminologie ne peut revendiquer un statut indépendant d'autres composantes du savoir; la terminologie est partie intégrante de la plupart des programmes d'enseignement et de formation le moindrement spécialisés; la terminologie ne peut assurer son avenir qu'en s'inscrivant sur une trajectoire qui croise la science informatique; la terminologie assume une fonction de communication qui est loin d'être négligeable; la terminologie doit intégrer en son sein et de manière plus explicite la dimension communicationnelle perçue sous l'angle variationniste. Les deux derniers éléments ont quelque chose à voir avec une socioterminologie en train de naitre (voir les Cahiers de linguistique sociale, $\mathrm{n}^{\circ} 18,1991$ ). Quoique PCTP prenne l'anglais comme lieu d'ancrage des affirmations et des démonstrations de son maître d'œuvre, son discours est presque universellement applicable à une langue ou à une autre, moyennant, bien entendu, quelques ajustements indispensables. Cet arrièreplan domine les huit chapitres qui servent d'armature au livre.

Il semble de mise de jeter un rapide coup d'œil circonstancié sur chacun des chapitres de PCTP.

Le chapitre introductif (pp. 1-12) définit la terminologie comme un nouveau champ de recherche et d'activités interdisciplinaires pour les langagiers ainsi que pour les linguistes, qui commencent à s'y intéresser de plus en plus sérieusement. J. C. Sager la conçoit comme un ensemble de pratiques qui ont évolué dans l'orbite d'un certain nombre de satellites telles la création des termes, la cueillette des unités spécialisées ainsi que l'explication sur leur contenu sémantique (notion/concept), leur mise en dictionnaire sous format traditionnel ou électronique, pour demeurer ici à l'intérieur des frontières de 1.a linguistique. La terminologie serait donc très précisément «the study of and the field of activity concerned with the collection, description, processing, and presentation of terms, i.e. lexical items belonging to specialised areas of usage of one or more languages» (p. 2). Par ailleurs, dans son usage actuel, le terme terminologie présente un profil polysémique qui recouvre au moins trois significations majeures:

1. c'est une activité pratique et méthodologique nécessaire à la collecte, la description et la présentation des termes;

2. c'est une théorie, c'est-à-dire un ensemble de prémisses, de raisonnements et de conclusions requis pour expliquer un réseau de relations entre les termes et les concepts;

3. c'est le vocabulaire d'une sphère d'activité déterminée, d'un discours thématique ( $L S P$ en anglais). 
Le reste de ce premier chapitre rappelle à propos que la terminologie entretient des rapports étroits avec d'autres disciplines d'ordre linguistique et extralinguistique, que nombre de linguistes ont une méconnaissance parfaite ou une conception limitée, voire erronée de la terminologie et qu'ils la ramènent à une spécialisation de la lexicologie/ -graphie, que les développements présents de la terminologie soulèvent une foule de problèmes conflictuels entre la théorie et la pratique, toutes assertions qu'on ne peut que confirmer de ce côté-ci de l'Atlantique et pour les recherches portant sur le français.

Le deuxième chapitre (pp. 13-54) est centré sur la dimension cognitive de la terminologie. Il met les formes linguistiques en rapport avec leur contenu conceptuel et leur référence à la réalité de l'univers (le référent dans la théorie du signe linguistique). Quatre sous-divisions sont l'occasion d'expliciter comment la théorie de la référence contribue à distinguer les termes ( $L S P$ ) et les mots (langue générale: LG). Cette dichotomie est avant tout d'ordre pratique mais néanmoins essentielle afin de tracer une ligne de démarcation utile, quoique imaginaire, entre le champ de la terminologie et celui de la lexicologie. Ce chapitre remet sur le gril de la critique ce qu'est un concept (notion dans la terminologie de l'ISO) tandis que différentes formules définitionnelles sont ravivées, notamment celles de l'ISO. L'auteur note également quelques autres éléments fondamentaux sur les concepts, comme leurs caractéristiques logiciennes, leurs types (catégories), les structures qui filent leurs relations. À l'image de la lexicographie, la définition est l'une des phases délicates, essentielles et critiques du travail de recherche terminologique/-graphique. Aussi paraît-il opportun d'en examiner attentivement les fondements, les classes, les buts et les interrelations tissées dans un même réseau conceptuel. Dans un ensemble terminologique structuré onomasiologiquement, un énoncé définitionnel n'est jamais isolé, autarcique. Il est étroitement dépendant de l'organisation du vocabulaire soumis à la description à des fins dictionnairiques. C'est d'abord par ce réseau, cette filière que les liens internotionnels s'établissent.

Le chapitre trois (pp. 55-97) introduit la dimension linguistique de la terminologie sous la forme des représentations lexicales existantes (les termes) et potentielles (la néologie) des terminologies en tant que vocabulaires. C'est donc une approche du terme considéré comme un élément linguistique (linguistic item) tel qu'il se présente dans le discours métalinguistique de toutes les formes de dictionnaires terminologiques qui est ici proposée. Tout en reconnaissant les liens entre la terminologie et la lexicologie/-graphie, J. C. Sager centre ses propos sur les seuls aspects de la lexicologie qui ont des incidences sur la production des outils-dictionnaires et sur les perceptions de l'usage, volets qu'il faut rapprocher de la normalisation. Trois sous-sections traitent successivement de la théorie du terme: 1. la démarche onomasiologique; 2 . 1'aspect formel des termes (leurs rapports aux dictionnaires, leurs structurations relationnelles, leurs statuts); la théorie et la pratique de la formation des mots dans toutes leurs dimensions car c'est là le moyen privilégié de faire passer le message terminologique (brève typologie de la création lexicale, emprunt, attitudes des usagers devant l'inédit linguistique); 3. les directives institutionnelles au sujet de la création des termes nouveaux, notamment les propositions de l'ISO, devenues plus ou moins obsolètes, sont passées en revue. Cette troisième section jette également un regard sur les systèmes nomenclaturaux relatifs à quelques phénomènes naturels comme les sciences biologiques, la géologie ( $\mathrm{y}$ inclus la minéralogie), la chimie et la médecine. Dans ces domaines, le besoin de classification et de classement par ordre est fondamental. Les caractères spécifiques des nomenclatures médicale, biologique et chimique sont brièvement notés et commentés.

Le chapitre quatre (pp. 99-128) inscrit la terminologie dans le cadre de la communication. Il s'agit d'observer ici comment les termes pénètrent et opèrent dans un modèle de communication donné. Cela suppose un rapport à l'usage socioprofessionnel qui affecte 
dans un sens ou dans l'autre la nature et l'emploi des unités thématiques ( $L S P)$. L'approche communicationnelle exige en outre que soient cernées les différentes catégories d'utilisateurs des complexes terminologiques mis en circulation, ainsi que l'usage qu'ils font des services offerts. L'auteur définit donc un modèle de communication dans la perspective où un encodeur envoie un message à un décodeur qui doit l'interpréter et le comprendre pour que la connaissance soit modifiée dans le sens de l'augmentation de l'information existante sur un sujet (à savoir l'accroissement des connaissances pour l'expert ou l'acquisition des premières connaissances pour l'apprenant en formation). Sager examine tous les paramètres extralinguistiques (l'intention, l'information ellemême par rapport au niveau de connaissance et du domaine...) qui précèdent l'envoi et la réception du message; ce dernier suppose lui-même le choix de la langue ou du souscode thématique reconnaissable par le destinataire (le didacticien ne parle pas de la même façon à ses pairs et à ses élèves). L'efficacité du message passe alors par l'efficacité des termes sélectionnés pour le véhiculer. D'où l'identification de critères d'économie, de précision et de convenance, ce dernier afin d'arbitrer les deux premiers qui pourraient s'opposer ou s'exclure. On concevra alors aisément que la mise en valeur du rôle de la communication permette de proposer d'une manière fort concrète une socioterminologie, en train de s'édifier par ailleurs.

Inévitablement, un modèle communicationnel débouche sur une sorte de réductionnisme linguistique dont la forme la plus achevée prend la couleur de la normalisation ou de la standardisation, dans le vocabulaire de l'ISO. Au dire de l'auteur, l'harmonisation linguistique équilibre les critères d'économie (choix du terme le plus représentatif du point de vue de la brièveté), de précision (sous-critère de la clarté, donc d'indice référentiel ou d'élimination de l'ambiguîté) et de convenance (dans le cas où des connotations négatives (disturbing) pourraient intervenir). Les aspects prescriptifs de la terminologie ont maintes fois été critiqués et remis en question, mais les solutions de remplacement furent plutôt rares, comme furent encore plus rares les propositions de rejet total d'une certaine forme de dirigisme, d'aménagement ou de prescriptivisme! Une critique plus constructive est développée ici, qui inclut les avantages et les désavantages de toute opération d'harmonisation de la communication. Afin de bien resituer les choses dans leur contexte véritable, Sager dissèque scrupuleusement les principes et les instruments de normalisation (dictionnaires, banques de termes, commissions ministérielles). Il veut faire saisir les motivations qui soutiennent la standardisation linguistique. C'est en réinterprétant les principes de l'ISO au regard de la standardisation des objets qu'il réaffirme ce qu'est la normalisation terminologico-linguistique. Les sept grands principes de l'organisme viennois sont ranimés et replacés sous l'éclairage des $L S P$. Bien entendu, l'objectif premier consiste à faciliter la communication afin que le message transmis ou à transmettre s'accompagne de la meilleure efficacité possible. Chaque principe peut être synthétisé en une brève formule:

- simplification de l'expression;

- recherche d'un consensus dans un domaine donné;

- implantation dans le discours;

- statut linguistique (caractères dérivationnels, compositionnels ou syntagmatiques);

- vérification de la fonctionnalité des normes;

- éclairage social (industrialisation, lois, etc.).

D'autres questions plus subsidiaires, comme les méthodes de standardisation, l'efficacité réelle et les limites des normes, terminent cette section, les exemples qui l'illustrent étant souvent puisés dans le réservoir des normes britanniques (BS). 
Ce que j'appellerais la seconde grande articulation du livre, soit les chapitres 5 à 7 , noue les liens de la terminologie avec la science informatique, perçue ici dans ses dimensions les plus englobantes. Chaque chapitre est consacré à un aspect spécifique du traitement de la terminologie à l'aide des différentes technologies informatiques disponibles.

Le chapitre cinq (pp. 129-162) porte sur la compilation des terminologies. Il est un fait admis que l'automatisation et, surtout, la micro-informatique ont révolutionné cette grande phase du travail de recherche terminologique. Pour J. C. Sager, «It is now recognised that the only practical means of processing lexical data is by computer» (p. 129). En conséquence, les références aux méthodes de manipulation plus traditionnelles de. viennent presque caduques. D'ailleurs, il n'y en a aucune dans ce chapitre. Au contraire, l'auteur préconise l'établissement de nouveaux principes et la modernisation des méthodes de l'époque de la pré-automatisation. Tout comme la lexicographie, la terminologie souffre beaucoup, en ce moment, de l'absence de tels fondements théoriques. Sager aborde la question de la constitution des corpus informatisés, celle des bases de données à caractère terminologique et celle des nouvelles technologies, logicielles notamment, ainsi que celle des corpus textuels directement accessibles en ordinateur. Le stockage ou le réemploi de l'information terminologique en ordinateur créent de nouvelles contraintes méthodologiques; ils amènent en outre à se pencher sur la qualité des données et à réexaminer les principes de la collecte des données. Toute information devrait être enregistrée suivant des critères qui permettent de dessiner un profil du terme et du concept décodable au premier degré; d'où le regard critique sur toutes les composantes d'une fiche informatisée (record).

Le chapitre six (pp. 163-186) discute du stockage des termes, plus particulièrement en référence avec les banques de termes, ce qui ne signifie pas que les principes décrits ne soient pas valables pour d'autres formes d'enregistrement, tant s'en faut. Une partie de ce chapitre est d'ordre historique, spécialement le rappel de l'émergence des banques dont l'origine se situe aux environs de 1970. Ce tour d'horizon par l'histoire amène l'auteur à proposer une définition-programme de ce que devrait être (est?) une banque, à savoir :

A collection, stored in a computer, of special language vocabularies, including nomenclatures, standardised terms and phrases, together with the information required for their indentification, which can be used as a mono- or multilingual dictionary for direct consultation, as a basis for dictionary of usage and term creation and as an ancillary tool in information and documentation (p. 169).

Puis, un modèle de banque est proposé, illustrant la définition ci-dessus, avant de déboucher, en fin de chapitre, sur les réseaux sémantiques mis à l'ordre du jour, en tant que nécessité, par la recherche en et sur l'intelligence artificielle.

Le septième chapitre (pp. 187-205) scrute le réemploi, l'implantation, les retombées de la terminologie. De fait, toute information consignée devrait pouvoir être traitée ou retraitée et/ou elle devrait pouvoir être accessible aux usagers potentiels. Sager présente les différentes figures de réemploi, leurs contraintes et le réagencement de l'information terminologique transmise ou à transmettre. Ce chapitre typologise les différents groupes d'utilisateurs des terminologies stockées, à savoir : les spécialistes de chaque discipline, les professionnels de la communication spécialisée (les médiateurs, les traducteurs...), les terminographes et les terminologues, les professionnels de la documentation et de l'information documentaire, les aménagistes, tout groupe qui s'occupe de questions linguistiques à des fins professionnelles (enseignants, éditeurs, rédacteurs, correcteurs), l'usager général. Le chapitre est clos par l'établissement d'une connexion avec les thésaurus terminologiques. 
Le dernier chapitre de ce livre (pp. 207-229) s'intéresse à l'application des terminologies, aux attitudes et aux pratiques qui existent. La croissance, le développement ainsi que les principales activités de traitement de la terminologie retiennent également l'attention. Ce chapitre se veut à la fois conclusif et ouvert sur l'avenir car il est quasi impossible de saisir dans son ensemble ce que sont la production et le traitement de la terminologie. Il n'y a pas d'application possible sans les quelques conditions que sont l'échange de l'information, la planification, la conscience de la variation de l'usage, la disponibilité des données et la mise à jour périodique, série de critères qui concrétisent la physionomie de plus en plus socioterminologique des LSP.

L'analyse de l'auteur le conduit à constater qu'à l'heure actuelle, il n'existe pas de position unique du point de vue théorique devant les arguments de principes et de méthodes de la terminologie. Cela s'explique historiquement lorsque l'on observe la grande variété des dictionnaires terminologiques produits, les structures variées des différentes banques de termes et, surtout, lorsqu'on en perçoit l'origine dans les programmes d'aménagement élaborés par les organismes ou les individus diatopiquement différents. Toute l'armada des décisions influence inévitablement les orientations des principes théoriques et méthodologiques.

Ce chapitre met aussi l'accent sur le rôle et l'historique des banques de terminologie. Une brève description des principales grandes banques est donnée et un aperçu des développements des petites banques montre bien l'impact de la micro-informatique. La miniaturisation a simultanément des avantages et des désavantages car, d'une part, elle rend les terminologies de plus en plus accessibles à de plus en plus de gens tandis que, d'autre part, leur développement est lent et fort peu de minibanques sont vraiment opérationnelles. Leur nombre fait qu'il n'y a pas de consensus méthodologique, pas de structures communes et par conséquent une limitation de leur consultation. Par ailleurs, plus leur nombre croîtra, plus ces préoccupations d'harmonisation seraient urgentes à analyser afin de trouver des solutions pour éviter le morcellement.

Une très bonne bibliographie thématique et sélective, listant quelques centaines de titres (390 exactement) regroupés en huit sous-catégories, termine l'ouvrage (pp. 231-254). Elle constitue une référence et un guide fort utile pour tout chercheur intéressé par un aspect ou un autre de la terminologie.

Ce livre fait donc le point sur un certain nombre de données fondamentales en terminologie à partir du principe que la terminologie d'aujourd'hui n'a guère de chances de se développer hors du champ de l'informatique. L'auteur fournit suffisamment de justificatifs pour qu'on prête foi à ses démonstrations. Il aura fallu moins de vingt ans pour que la terminologie établisse un corps de doctrine multidisciplinaire et quasi universel et qu'elle accède au statut d'une technologie utile en sciences humaines. Déjà, dès les années 1930, Wüster en explicitait les composantes extralinguistiques auxquelles il greffait l'informatique au début des années 1970. Une toute petite génération informatique avec en son centre la poussée de la micro-informatisation - aura suffi à fusionner les deux éléments fondamentaux qui ont servi à J. C. Sager pour structurer son cours pratique sur la recherche et le traitement de la terminologie.

Dans le carrousel de la production écrite sur la science terminologique, ce livre, dont la matière est fort bien équilibrée et accessible au néophyte, comptera comme l'une des très bonnes sommes existantes, riche d'enseignement et dont la carrière universitaire est assurée en raison de la validité des informations qu'il recèle. Ce genre de livre de chevet est rare. Nombre de ses semblables ont vieilli prématurément et se sont fossilisés. Ce ne sera pas le cas de PCTP qui va au cour même des fondements historiques, actuels et universels de la terminologie. Voilà un bon, un véritable guide pour l'apprenant et pour l'expert, un manuel agrémenté de nombreux exemples, ce qui ne nuit jamais au décodage 
d'un texte et fait le pont avec le réel. À ranger à portée de main dans sa bibliothèque, au même titre que par exemple Rey et Kocourek, pour ne citer que des ouvrages accessibles en français. Telle pourrait éventuellement être la carrière du livre de Sager.

JEAN-ClAUde BOULANGER Université Laval, Québec, Canada 